\title{
Estabilidade de sistemas lineares chaveados incertos por meio de funções de Lyapunov estruturadas *
}

\author{
Yan Teixeira Felber* Natália Augusto Keles* Márcio Júnior Lacerda* \\ * GCOM - Grupo de Controle e Modelagem \\ Departamento de Engenharia Elétrica \\ UFSJ - Universidade Federal de São João del-Rei \\ Pça. Frei Orlando, 170 - Centro - 36307-352 - São João del-Rei, MG, Brasil \\ (e-mails: yanfelber@gmail.com,natalia.augusto.keles@hotmail.com, \\ lacerda@ufsj.edu.br).
}

\begin{abstract}
This paper is concerned with the stability problem of uncertain linear switched systems. The main contribution is to provide a systematic procedure to check the stability of discrete-time uncertain switched systems by using Lyapunov functions with an augmented state vector. The formulation presented in the paper makes use of an augmented Lyapunov matrix that also can be considered in a block diagonal form. The sufficient conditions are given in terms of Linear Matrix Inequalities that can be solved by using available computational packages. Numerical examples from the literature illustrate the performance of the proposed method when compared with other approaches available in the literature.

Resumo: Este artigo trata do problema de estabilidade de sistemas lineares chaveados incertos. A principal contribuição é a apresentação de um procedimento sistemático para verificar a estabilidade de sistemas chaveados incertos discretos no tempo, usando funções de Lyapunov com um vetor de estados aumentados. A formulação apresentada faz uso de uma matriz aumentada de Lyapunov que também pode ser considerada na forma bloco diagonal. As condições suficientes são dadas na forma de desigualdades matriciais lineares que podem ser resolvidas usando pacotes computacionais existentes. Os exemplos numéricos retirados da literatura ilustram o desempenho do método proposto quando comparado a outras técnicas disponíveis na literatura.
\end{abstract}

Keywords: Switched systems; polytopic uncertainties; Lyapunov theory; stability analysis; uncertain systems.

Palavras-chaves: Sistemas chaveados; incertezas politópicas; teoria de Lyapunov; análise de estabilidade; sistemas incertos.

\section{INTRODUÇÃO}

Sistemas chaveados são uma classe de sistemas híbridos compostos por um determinado número de subsistemas em que cada um deles pode estar ativo individualmente (Daafouz et al., 2002; Dehghan e C. Ong, 2012) a cada instante de tempo. Para ativar cada subsistema é necessário uma regra de chaveamento que pode ser dependente do estado, do tempo, ou ainda ser autônoma (Liberzon, 2003). No chaveamento autônomo (ou arbitrário), supõe-se uma situação que não há controle direto sobre os mecanismos de comutação (Liberzon, 2003). Sistemas chaveados tem recebido grande atenção nas últimas décadas, em que autores como Liberzon e Morse (1999), Daafouz et al. (2002), Bertolin et al. (2018) e Gomide e Lacerda (2018) propuseram condições para certificar a estabilidade desses sistemas, quando submetidos ao chaveamento arbitrário. Também podem ser encontrados na literatura, estudos que apresentam condições para o projeto de regras de chaveamento que garantam a estabillidade dos sistemas (Hespanha e Morse, 1999; Zhai et al., 2000).

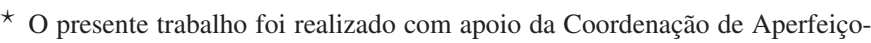
amento de Pessoal de Nível Superior - Brasil (CAPES) - Código de Financiamento 001, FAPEMIG APQ-00692-17, CNPq 425800/2018-0 e PPGEL-UFSJ.
}

Tendo em vista a ampla aplicabilidade dos sistemas chaveados, existe espaço na literatura de controle, para novos estudos que tenham como objetivo certificar a estabilidade e projetar regras de chaveamento mais eficientes e menos conservadoras. Nesse sentido, a Teoria de Lyapunov (Khalil, 2002) é uma forte aliada, pois possibilita obter as condições na forma de Desigualdades Matriciais Lineares (LMIs, do inglês Linear Matrix Inequalities) que são uma ferramenta matemática utilizada para solucionar problemas com formulações convexas, muito comuns na área de sistemas de controle, além de prover metodologias muito consistentes para modelos na presença de incertezas (Boyd et al., 1994).

$\mathrm{Na}$ literatura, pode-se encontrar inúmeros exemplos da Teoria de Lyapunov aplicada em sistemas chaveados, como em Daafouz et al. (2002), que propôs uma função de Lyapunov chaveada para análise de estabilidade de sistemas chaveados discretos no tempo sob chaveamento arbitrário, além disso, uma estratégia para projetar um controlador estático de saída foi apresentada. Em Lee e Dullerud (2006), uma condição para certificar a estabilidade de sistemas chaveados discretos foi apresentada considerando uma função de Lyapunov dependente do caminho. Hetel et al. (2006b) investigou o problema de estabilização para sistemas chaveados considerando atrasos variantes no tempo e Jungers et al. (2017) apresentou diferentes conjuntos 
de LMIs que podem ser usados para certificar a estabilidade de sistemas chaveados discretos no tempo. Recentemente, novas técnicas baseadas no emprego de funções de Lyapunov aumentadas foram introduzidas (Bertolin et al., 2018; Gomide e Lacerda, 2018). Essa nova classe de funções é capaz de certificar a estabilidade assintótica de sistemas chaveados usando um menor número de variáveis do que outros métodos presentes na literatura.

Mesmo com um crescente número de trabalhos focados na análise de estabilidade para sistemas chaveados, uma pequena parte desses trabalhos considera a presença de incertezas nos subsistemas. Desse modo, ao analisarmos estes sistemas é importante considerar a presença e o efeito das incertezas na análise de estabilidade (Binazadeh e Bahmani, 2017, 2016; Binazadeh e Shafiei, 2014). É possível encontrar na literatura diferentes abordagens para a representação de incertezas, entre elas pode-se citar as incertezas politópicas (Kermani e Sakly, 2014; Niamsup e Rajchakit, 2013; Rajchakit et al., 2012), as incertezas limitadas em norma (Zhang e Yan, 2015; Sun et al., 2006) e as incertezas na forma afim (Baleghi e Shafiei, 2018). Dentre as três, as incertezas politópicas tendem a apresentar modelos menos conservadores quando comparados com outras formas de incertezas (Baleghi e Shafiei, 2018).

Este trabalho tem como objetivo propor novas condições para análise de estabilidade de sistemas chaveados incertos discretos no tempo sobre regra de chaveamento arbitrário. Cada subsistema incerto será considerado como pertencente a um domínio politópico. A estabilidade do sistema será certificada com base em uma função de Lyapunov estruturada composta por um vetor de estados aumentado. A escolha desta função permite introduzir a dinâmica do sistema chaveado e a incerteza politópica na função de Lyapunov. Desta forma, a função empregada é uma função de Lyapunov chaveada submetida a incertezas politópicas. Esse método é baseado na função de Lyapunov apresentada em Gomide e Lacerda (2018) para sistemas chaveados e que também foi explorada em (Pessim et al., 2018, 2019) para sistemas incertos. Assim, como objetivo principal do trabalho, foi proposto um método mais eficiente de garantir a estabilidade de um sistema chaveado submetido a incertezas politópicas. Dois exemplos numéricos são apresentados para comparação dos resultados com outras técnicas já difundidas na literatura.

O artigo é organizado da seguinte forma. A Seção 2 introduz os resultados preliminares. Os resultados principais são descritos na Seção 3. A Seção 4 apresenta os experimentos numéricos que ilustram o desempenho do método proposto e a Seção 5 conclui o artigo.

\section{RESULTADOS PRELIMINARES}

\subsection{Descrição do sistema}

Considere o seguinte sistema chaveado discreto no tempo submetido a incerteza politópica.

$$
x(k+1)=A_{\sigma(k)}(\alpha) x(k)
$$

em que $x \in \mathbb{R}^{n}$ é o vetor de estados e $A_{\sigma(k)}(\alpha) \in \mathbb{R}^{n \times n}$ é a matriz dinâmica do sistema. $\sigma(k)$, pertence a um conjunto finito $\mathscr{P}=\{1, \cdots, m\}$, que denota a regra de chaveamento. $\alpha$ pertence a um domínio politópico parametrizado em termos de um vetor de parâmetros invariantes no tempo. Para um modo $\sigma(k)$ é possível escrever

$$
A_{\sigma(k)}(\alpha)=\sum_{i=1}^{v} \alpha_{i} A_{\sigma(k) i}, \quad \alpha \in \Lambda_{v}
$$

em que $A_{\sigma(k) i}, i=1, \ldots, v$, são os vértices do politopo e $\Lambda_{v}$ é o simplex unitário

$$
\Lambda_{v}=\left\{\alpha \in \mathbb{R}^{v}: \sum_{i=1}^{v} \alpha_{i}=1 ; \alpha_{i} \geq 0, i=1, \ldots, v\right\}
$$

Como apenas um modo da matriz $A_{\sigma(k)}$ pode estar ativo por vez, o modo ativo será representado usando uma função indicadora. Seja $\xi(k)=\left[\xi_{1}(k), \cdots, \xi_{m}(k)\right]^{T}$

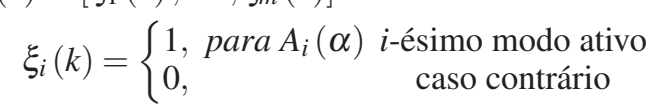

Dessa forma o sistema (1) pode ser escrito de forma geral como

$$
x(k+1)=A(\xi(k), \alpha) x(k)
$$

\subsection{Análise de estabilidade}

A estabilidade do sistema (1) pode ser certificada por meio de uma função de Lyapunov radialmente ilimitada $V(k, x(k))$. uma vez que o problema pode ser otimizado ao obter-se condições escritas na forma de desigualdades matriciais lineares. Para tal, uma função de Lyapunov deve satisfazer os seguintes critérios (Vidyasagar, 1993):

$$
\begin{gathered}
V(k, x(k))>0 \quad \forall x \neq 0 \\
\Delta V(k, x(k))<0 \quad \forall x \neq 0 \\
V(k, 0)=0
\end{gathered}
$$

Em Daafouz et al. (2002) foi proposta a seguinte função quadrática de Lyapunov $V(k, x(k))=x(k)^{T} P(\xi(k)) x(k)$ para certificar a estabilidade de sistemas chaveados discretos no tempo. O primeiro Lema propõe o uso dessa função na análise da estabilidade de um sistema com uma matriz $A$ chaveada e incerta:

Lema 1. Se existirem matrizes definidas positivas $P_{i} \in \mathbb{R}^{n \times n}$ $\forall i \in\{1, \ldots, m\}$ tais que

$$
A_{i}(\alpha)^{T} P_{j} A_{i}(\alpha)-P_{i}<0
$$

$\forall \alpha \in \Lambda_{v}, i \in\{1, \ldots, m\}$ e $j \in\{1, \ldots, m\}$, então, o sistema (2) é Globalmente Uniformemente Assintoticamente Estável (GUAS, do inglês Globally Uniformly Asymptotically Stable).

Prova. Pré e pós multiplicando a função indicadora apresentada em (6) por $x(k)^{T}$ e $x(k)$ tem-se

$$
\begin{aligned}
x(k)^{T} A(\xi(k), \alpha)^{T} P(\xi(k+1)) & A(\xi(k), \alpha) x(k) \\
& -x(k)^{T} P(\xi(k)) x(k)<0
\end{aligned}
$$

usando (2) em (7) obtém-se

$$
x(k+1)^{T} P(\xi(k+1)) x(k+1)-x(k)^{T} P(\xi(k)) x(k)<0
$$

Em que a condição (8) é equivalente a $\Delta V(k, x(k))<0$ para $V(k, x(k))=x(k)^{T} P(\xi(k)) x(k) \operatorname{com} V(k, x(k))>0$, assim conclui-se a prova.

Em Hetel et al. (2006a), foram introduzidas condições para a análise da estabilidade robusta de sistemas chaveados em que cada modo do sistema é descrito por um conjunto politópico representado em função de um vetor de parâmetros variantes 
no tempo. O Lema apresentado na sequência é uma adaptação de (Hetel et al., 2006a, Teorema 3) para o caso tratado nesse artigo, i.e., incertezas invariantes no tempo.

Lema 2. Se existirem matrizes simétricas definidas positivas $P_{i}(\alpha) \in \mathbb{R}^{n \times n}$ e $\forall i \in\{1, \ldots, m\}$ tais que

$$
A_{i}(\alpha)^{T} P_{j}(\alpha) A_{i}(\alpha)-P_{i}(\alpha)<0
$$

$\forall \alpha \in \Lambda_{v}, i \in\{1, \ldots, m\}$ e $j \in\{1, \ldots, m\}$, então o sistema (2) é GUAS.

Prova. A prova segue os mesmos passos da prova apresentada no Lema 1, usando uma função de Lyapunov com $P$ chaveada e incerta expressa por $V(k, x(k))=x(k)^{T} P(\xi(k), \alpha) x(k)$.

Diferentemente de (Hetel et al., 2006a, Teorema 3), o Lema 2 não faz uso de variáveis de folga.

\section{RESULTADOS PRINCIPAIS}

A elaboração de um conjuntos de LMIs capazes de certificar a estabilidade de sistemas lineares chaveados e incertos por meio de funções de Lyapunov estruturadas, é o resultado principal deste trabalho. Essa nova classe de funções de Lyapunov considera a dinâmica do próprio sistema em sua construção. Antes de apresentar o caso genérico, faz-se necessário exemplificar o desenvolvimento por meio de um caso particular dessa nova classe de funções. Considere a função de Lyapunov apresentada a seguir

$$
V(k, x(k))=\theta^{T}\left[\begin{array}{cc}
P_{1} & 0 \\
0 & P_{2}
\end{array}\right] \theta
$$

com

$$
\theta^{T}=\left[x(k)^{T} x(k+1)^{T}\right]
$$

Uma condição suficiente para a análise de estabilidade do sistema (2) é apresentada na sequência.

Lema 3. Se existirem matrizes simétricas $P_{1} \in \mathbb{R}^{n \times n}$ e $P_{2} \in$ $\mathbb{R}^{n \times n}$ tais que

$$
P_{1}+A_{i}(\alpha)^{T} P_{2} A_{i}(\alpha)>0
$$

$$
\begin{aligned}
A_{i}(\alpha)^{T} P_{1} A_{i}(\alpha)+A_{i}(\alpha)^{T} A_{j}(\alpha)^{T} P_{2} A_{j}(\alpha) A_{i}(\alpha) \\
-\left(P_{1}+A_{i}(\alpha)^{T} P_{2} A_{i}(\alpha)\right)<0
\end{aligned}
$$

$\forall \alpha \in \Lambda_{v}, i \in\{1, \ldots, m\}$ e $j \in\{1, \ldots, m\}$, então o sistema (2) é GUAS.

Prova. Pré e pós multiplicando (13) por $x(k)^{T}$ e $x(k)$ tem-se

$$
\begin{aligned}
& x(k)^{T} A(\xi(k), \alpha) P_{1} A(\xi(k), \alpha) x(k)+\Upsilon^{T} P_{2} A \Upsilon \\
& -\left(x(k)^{T} P_{1} x(k)+x(k)^{T} A(\xi(k), \alpha) P_{2} A(\xi(k), \alpha) x(k)\right)<0
\end{aligned}
$$

com

$$
\Upsilon=A(\xi(k+1), \alpha) A(\xi(k), \alpha) .
$$

usando (2) e substituindo em (14) obtém-se

$$
\begin{aligned}
& x(k+1)^{T} P_{1} x(k+1)+x(k+2)^{T} P_{1} x(k+2) \\
& -\left(x(k)^{T} P_{1} x(k)-x(k+1)^{T} P_{1} x(k+1)\right)<0
\end{aligned}
$$

A condição (16) é equivalente $\Delta V(k, x(k))<0$, definindo

$$
V(k, x(k))=x(k)^{T} P_{1} x(k)+x(k+1)^{T} P_{2} x(k+1)
$$

ou de maneira similar

$$
\begin{aligned}
& V(k, x(k))=x(k)^{T} P_{1} x(k) \\
& +x(k)^{T} A(\xi(k), \alpha)^{T} P_{2} x(k+1) A(\xi(k), \alpha) x(k)
\end{aligned}
$$

Assim, conclui-se a prova.

Observe que, mesmo fazendo uso de matrizes $P_{1}$ e $P_{2}$ constantes, a função de Lyapunov em (18) é chaveada e incerta. Além disso, as matrizes $P_{1}$ e $P_{2}$ são simétricas e não necessariamente definidas positivas. Para a condição proposta no Lema 3, um caso particular foi considerado com um vetor de estados aumentado definido como $N=2$ contendo $x(k)$ e $x(k+N-1)$. Para o caso geral, serão considerados $N$ vetores de estado. A função de Lyapunov assumirá a seguinte forma.

$$
V(k, x(k))=\Psi^{T}\left[\begin{array}{cccc}
P_{1} & 0 & \cdots & 0 \\
0 & P_{2} & \cdots & 0 \\
\vdots & \vdots & \ddots & \vdots \\
0 & 0 & \cdots & P_{N}
\end{array}\right] \Psi
$$

com

$$
\Psi=\left[\begin{array}{c}
x(k) \\
x(k+1) \\
\vdots \\
x(k+(N-1))
\end{array}\right]
$$

Teorema 4. Se existirem matrizes simétricas $P_{1}, \ldots, P_{N} \in \mathbb{R}^{n \times n}$ tais que

$$
\begin{gathered}
\sum_{z=1}^{N}\left[\prod_{\beta=0}^{z-1} A_{i_{\beta}}(\alpha)^{T} P_{z} \prod_{\beta=0}^{z-1} A_{i_{z-\beta-1}}(\alpha)\right]>0 \\
\forall\left(i_{1}, \ldots, i_{N-1}\right) \in \mathscr{P}^{N-1} \\
\sum_{z=1}^{N}\left[\prod_{\beta=0}^{z-1} A_{i_{1+\beta}}(\alpha)^{T} P_{z} \prod_{\beta=0}^{z-1} A_{i_{z-\beta}}(\alpha)\right] \\
-\sum_{z=1}^{N}\left[\prod_{\beta=0}^{z-1} A_{i_{\beta}}(\alpha)^{T} P_{z} \prod_{\beta=0}^{z-1} A_{i_{z-\beta-1}}(\alpha)\right]<0 \\
\forall\left(i_{1}, \ldots, i_{N-1}\right) \in \mathscr{P}^{N-1}
\end{gathered}
$$

com $A_{i_{0}}(\alpha)=I$, então, o sistema (2) é GUAS.

Prova. A prova do Teorema 4 segue os mesmos passos da prova do Lema 3 considerando a função de Lyapunov como em (19).

Para um sistema chaveado precisamente conhecido, as condições do Teorema 4 recuperam as condições apresentadas em (Gomide e Lacerda, 2018, Teorema 5) que são equivalentes às condições apresentadas em (Bertolin et al., 2018, Teorema 4-ii)). Em Bertolin et al. (2018) foi demonstrado que para sistemas chaveados precisamente conhecidos, as soluções são necessárias e suficientes para um valor arbitrário $N$.

Todas as condições apresentadas até aqui, estão representadas na forma de LMIs robustas que dependem do parâmetro $\alpha$. Para obter um conjunto finito de LMIs em termos dos vértices de cada modo do sistema chaveado, o ROLMIP (Agulhari et al., 2012) foi empregado. O ROLMIP emprega estruturas politópicas e relaxações polinomiais (Oliveira e Peres, 2007) para obter um conjunto finito de LMIs. 


\section{EXPERIMENTOS NUMÉRICOS}

Nessa seção é apresentado um comparativo entre as condições propostas neste artigo com outras presentes na literatura. As rotinas foram implementadas em Matlab versão R2015a, utilizando pacotes computacionais YALMIP Löfberg (2004), SeDuMi F. (1999) e ROLMIP Agulhari et al. (2012). O computador utilizado possui um processador Intel Core i7 7500, 2.7 Ghz, 8 Gb de RAM com sistema operacional Windows 10.

\subsection{Exemplo 1}

Considere o sistema chaveado e incerto retirado de Hetel et al. (2006a). Nesse artigo, os parâmetros são considerados invariantes no tempo.

$$
\begin{aligned}
& x(k+1)=\hat{A}_{\sigma} x(k) \\
& \hat{A}_{\sigma}(k)=A_{0 \sigma}+D_{\sigma} F(k) E_{\sigma} \\
& \text { Assim, } \operatorname{com} F(k)=\rho(k), \quad F(k) \in[-1,1] \\
& A_{\sigma 1}=A_{0 \sigma}+\rho D_{\sigma} E_{\sigma} \\
& A_{\sigma 2}=A_{0 \sigma}-\rho D_{\sigma} E_{\sigma} \\
& A_{01}=\left[\begin{array}{ccccc}
0.2 & 0.2 & 0.3 & 0.1 & -0.5 \\
0.8 & 0 & -0.1 & -0.3 & 0.3 \\
0 & -0.3 & -0.4 & 0 & 0 \\
0 & 0.3 & 0.3 & 0.3 & 0.5 \\
-0.2 & 0 & 0 & 0 & 0.1
\end{array}\right] \\
& A_{02}=\left[\begin{array}{ccccc}
-0.7 & -0.7 & 0 & 0 & 0.2 \\
0.5 & 0.3 & 0.3 & -0.3 & 0 \\
0.3 & 0.4 & 0.3 & 0.6 & 0.3 \\
0.3 & -0.8 & 0 & 0 & 0 \\
0.1 & -0.7 & 0.1 & -0.3 & 0.3
\end{array}\right] \\
& \sigma \in[1,2] \\
& D_{1}^{T}=\left[\begin{array}{lllll}
0.2 & 0.5 & -0.1 & 0.3 & 0.2
\end{array}\right] \\
& D_{2}^{T}=\left[\begin{array}{lllll}
-0.5 & 0.38 & 0.5 & 0.2 & 0.5
\end{array}\right] \\
& E_{1}=\left[\begin{array}{lllll}
-0.3 & -0.3 & -0.5 & 0.2 & 0.3
\end{array}\right] \\
& E_{2}=\left[\begin{array}{lllll}
-0.2 & 0.1 & -0.1 & -0.05 & 0.7
\end{array}\right]
\end{aligned}
$$

Assim, o sistema chaveado e incerto pode ser descrito da seguinte forma:

$$
\begin{aligned}
& A_{1}(\alpha)=\alpha_{11} A_{11}+\alpha_{12} A_{12} \\
& A_{2}(\alpha)=\alpha_{21} A_{21}+\alpha_{22} A_{22}
\end{aligned}
$$

A Tabela 1 apresenta o número de variáveis de decisão escalares $\left(N_{V}\right)$ e o número de linhas de LMIs $\left(N_{L}\right)$ envolvidas no problema de otimização. Foram comparadas as condições do Lema 2 (adaptação de (Hetel et al., 2006a) sem variáveis de folga), adaptação de (Hetel et al., 2006a, Teorema 3) (Teo3Het - com variáveis de folga) e Lema 3. Todos os métodos testados foram capazes de certificar a estabilidade do sistema.

Tabela 1. Número de variáveis escalares $N_{V}$ e número de linhas de LMIs $N_{L}$ necessários para certificar a estabilidade do Exemplo 1, considerando diferentes métodos.

\begin{tabular}{ccc} 
Método & $N_{V}$ & $N_{L}$ \\
\hline Lema 2 & 60 & 100 \\
Teo3Het & 160 & 160 \\
Lema 3 & 30 & 130 \\
\hline
\end{tabular}

Os resultados para o Lema 1 não são apresentados, uma vez que para $\rho=1$ a solução é infactível. O Lema 2 é uma adaptação de (Hetel et al., 2006a, Teorema 3) em que, para esse caso, não foram utilizadas as variáveis de folga $G$ e $S$, principal motivo pelo qual $N_{V}$ e $N_{L}$ são distintos entre o Lema 2 e o Teorema 3 retirado de Hetel et al. (2006a).

O Lema 3, quando comparado com os demais métodos, apresentou maior eficência, sendo capaz de certificar a estabilidade do sistema com um menor número de variáveis escalares e um menor número de linhas de LMIs quando comparado ao método proposto por Hetel et al. (2006a).

A Figura 1 apresenta a evolução da função de Lyapunov, obtida por meio do Lema 3, ao longo das iterações e a regra de chaveamento empregada. Para a simulação, foi considerado chaveamento arbitrário, condição inicial $x(0)=\left[\begin{array}{lllll}1 & 2 & 3 & 4\end{array}\right]^{T}$ e $\alpha_{11}=0.1, \alpha_{12}=0.9, \alpha_{21}=0.9$ e $\alpha_{22}=0.1$. Considerando $V(x(k))$ como em (10), ou de maneira similar $V(x(k))=$ $V_{1}(x(k))+V_{2}(x(k)) \operatorname{com} V_{1}(x(k))=x(k)^{T} P_{1} x(k)$ e $V_{2}(x(k))=$ $x(k)^{T} A_{i}(\alpha)^{T} P_{2} A_{i}(\alpha) x(k)$ é possível verificar por meio da Figura 1 que o comportamento de $V(x(k))$ é monotonicamente decrescente, mesmo que as funções $V_{1}\left(x(k)\right.$ e $V_{2}(x(k))$ não sejam, o que mostra a consistência do método.

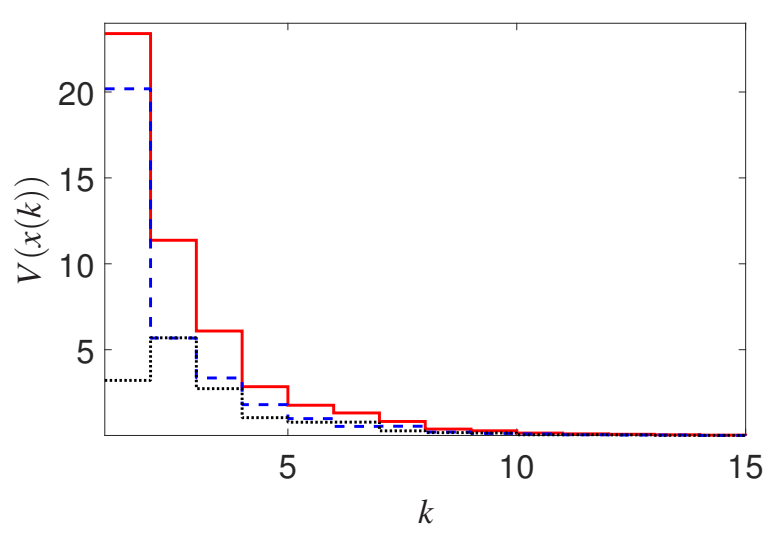

(a)

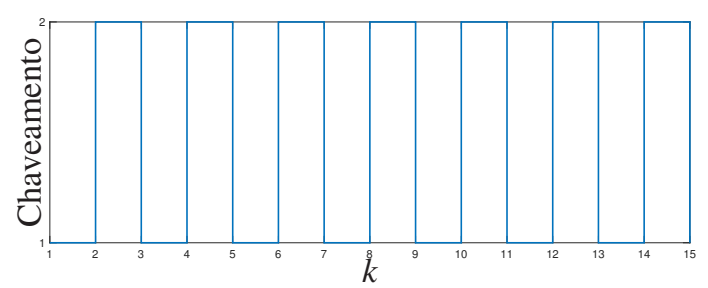

(b)

Figura 1. Função de Lyapunov obtida por meio do Lema 3. (a) A linha vermelha (contínua) expressa $V(x(k))$ como em (10), A linha azul (tracejada) apresenta a evolução de $V_{1}(x(k))$ e a linha preta (pontilhada) mostra a evolução de $V_{2}(x(k))$. (b) Regra de chaveamento empregada em função de $k$.

\subsection{Exemplo 2}

O segundo exemplo foi adaptado de Lee e Dullerud (2006). Seja o sistema chaveado e incerto, em que $\beta$ agora é um intervalo representado por $\beta \in[-\Theta, \Theta]$

$$
A_{1}(\alpha)=\left[\begin{array}{ll}
\beta & \beta \\
0 & 0
\end{array}\right], \quad A_{2}(\alpha)=\left[\begin{array}{cc}
-\beta & 0 \\
\beta & -\beta
\end{array}\right]
$$


O objetivo deste exemplo é encontrar o maior valor de $\Theta$ para o qual ainda é possível certificar a estabildade do sistema. A estratégia utilizada é baseada no Teorema 4 considerando diferentes valores de $N$. A Tabela 2 apresenta os valores para o limitante do intervalo $\beta$ bem como o número de variáveis escalares $N_{V}$ e número de linhas de LMIs $N_{L}$.

Tabela 2. Limitante de $\Theta$, número de variáveis escalares $N_{V}$ e número de linhas de LMIs $N_{L}$.

\begin{tabular}{cccccc}
\hline$N$ & 2 & 3 & 4 & 5 & 6 \\
$\Theta$ & 0.7413 & 0.7688 & 0.7805 & 0.7878 & 0.7913 \\
$N_{V}$ & 6 & 9 & 12 & 15 & 18 \\
$N_{L}$ & 52 & 152 & 400 & 992 & 2368 \\
\hline
\end{tabular}

Ao utilizar o Lema 2 e a técnica (Hetel et al., 2006a, Teorema 3) adaptada para o caso incerto, encontra-se que o valor limite de $\Theta$ para o qual ainda é possível certificar a estabilidade do sistema, para esse caso $\Theta=0.7548$. Usando o (Hetel et al., 2006a, Teorema 3) a estabilidade foi certificada com $N_{V}=28$ e $N_{L}=64$.Por sua vez, usando o Lema 2 a estabilidade é garantida com $N_{V}=12$ e $N_{L}=40$. Desse modo é possível observar que existe um ganho de eficiência para certificar a estabilidade desse sistema com o uso do Teorema 4. Também foi possível verificar que ao aumentar a ordem da função de Lyapunov descrita em (20) obteve-se valores maiores para os limitantes $\beta$. Esses valores quando comparados aos valores descritos em Lee e Dullerud (2006) são ligeiramente menores. Essa divergência de valores ocorre devido ao sistema tratado no Exemplo 2 ser incerto e chaveado.

A seguir são apresentadas as simulações temporais para o sistema do Exemplo 2 com $\beta \in[-0.7913,0.7913]$ (pior caso). A Figura 2 apresenta simulações para condições iniciais geradas aleatoriamente e valores de $\alpha$ fixos. Todos os sistemas testados, são estáveis, como esperado. A Figura 3 mostra a resposta temporal do sistema para a condição inicial $x(0)=\left[\begin{array}{ll}1 & 2\end{array}\right]^{T}$, e diferentes valores para $\alpha$. Foram considerados valores de $\alpha_{11}$ no intervalo [0,1], com $\alpha_{12}=1-\alpha_{11}, \alpha_{22}=\alpha_{11}$ e $\alpha_{21}=\alpha_{12}$. Os dois estados convergem para a origem ao longo das iterações.

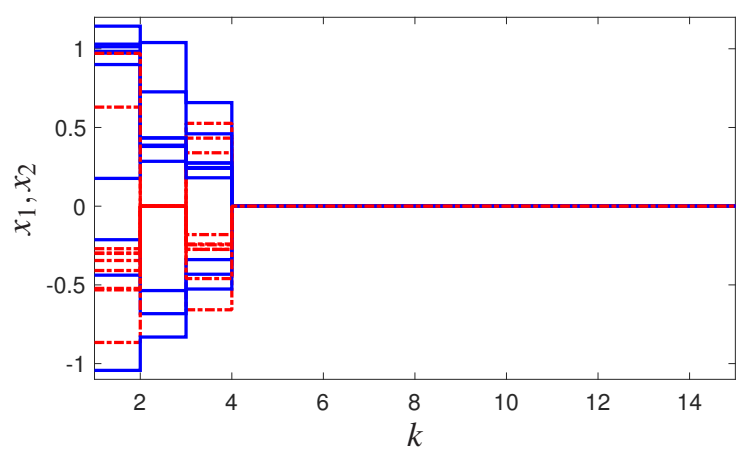

Figura 2. Evolução temporal do sistema, a linha azul (contínua) representa o primeiro estado e a linha vermelha (traço ponto) representa o segundo estado. Foram consideradas diferentes condições iniciais e os seguintes valores para os parâmetros incertos: $\alpha_{11}=0.1, \alpha_{12}=0.9, \alpha_{21}=0.9$ e $\alpha_{22}=0.1$.

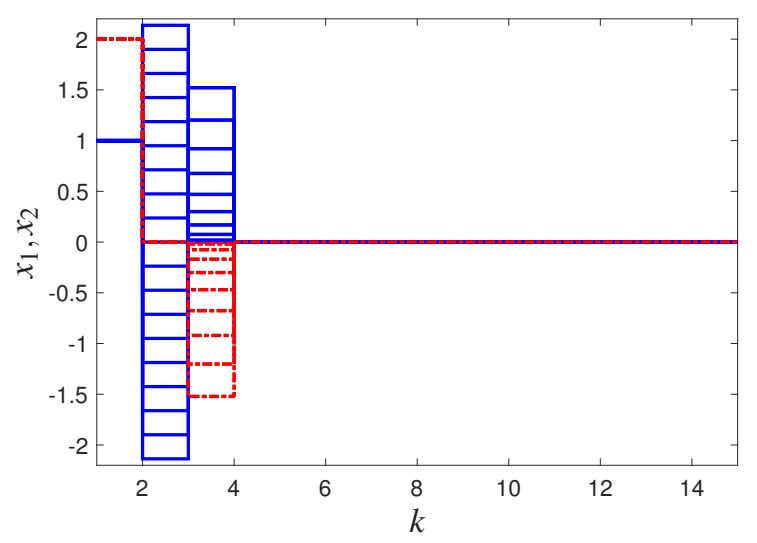

Figura 3. Evolução temporal do sistema, a linha azul (contínua) representa o primeiro estado e a linha vermelha (traço ponto) representa o segundo estado. Foram considerados $\alpha_{11}=[0,0.05, \ldots, 0.95,1], \alpha_{12}=1-\alpha_{11}, \alpha_{22}=\alpha_{11} \mathrm{e}$ $\alpha_{21}=\alpha_{12}$.

\section{CONCLUSÃO}

Este artigo apresentou novas condições na forma de LMIs para certificar a estabilidade de sistemas chaveados incertos discretos no tempo, por meio da Teoria de Lyapunov. A função de Lyapunov utilizada emprega um vetor de estados aumentado que é responsável por introduzir a dinâmica do próprio sistema na função de Lyapunov. As vantagens do método proposto foram demonstradas por meio de exemplos numéricos, em que o número de variáveis necessárias para certificar a estabilidade dos sistemas chaveados incertos foi menor que o utilizado por outras técnicas presentes na literatura. É possível concluir também que ao aumentar o valor de $N$, a função de Lyapunov proposta é capaz de certificar a estabilidade de matrizes chaveadas e incertas com incertezas maiores. Como extensão, para trabalhos futuros, os autores pretendem considerar a presença de parâmetros variantes no tempo.

\section{REFERÊNCIAS}

Agulhari, C.M., de Oliveira, R.C.L.F., e Peres, P.L.D. (2012). Robust LMI Parser: a computational package to construct LMI conditions for uncertain systems. In XIX Brazilian Conference on Automation (CBA 2012), 2298-2305. Campina Grande, PB, Brazil.

Baleghi, N.A. e Shafiei, M.H. (2018). Stability analysis for discrete-time switched systems with uncertain time delay and affine parametric uncertainties. Transactions of the Institute of Measurement and Control, 40(4), 1150-1157.

Bertolin, A.L.J., Oliveira, R.C.L.F., de Oliveira, M.C., e Peres, P.L.D. (2018). LMI-based stability tests for LPV and switched discrete-time linear systems through redundant equations. In Joint 9th IFAC Symposium on Robust Control Design and 2nd IFAC Workshop on Linear Parameter Varying Systems, 531-536. Florianópolis, SC, Brazil.

Binazadeh, T. e Bahmani, M. (2017). Design of robust controller for a class of uncertain discrete-time systems subject to actuator saturation. IEEE Transactions on Automatic Control, 62(3), 1505-1510.

Binazadeh, T. e Shafiei, M. (2014). Robust stabilization of uncertain nonlinear slowly-varying systems: Application in a time-varying inertia pendulum. ISA Transactions, 53(2), 373-379. 
Binazadeh, T. e Bahmani, M. (2016). Robust time-varying output tracking control in the presence of actuator saturation. Transactions of the Institute of Measurement and Control, 40(3), 61-70.

Boyd, S., Ghaoui, L.E., Feron, E., e Balakrishnan, V. (1994). Linear Matrix Inequalities in System and Control Theory. SIAM Studies in Applied Mathematics, Philadelphia, PA.

Daafouz, J., Pierre, R., e Iung, C. (2002). Stability analysis and control synthesis for switched systems: a switched Lyapunov function approach. IEEE Transactions on Automatic Control, 47(11), 1883-1887.

Dehghan, M. e C. Ong, C.j. (2012). Automatica discrete-time switching linear system with constraints : Characterization and computation of invariant sets under dwell-time consideration. Automatica, 48(5), 964-969.

F., S.J. (1999). Using sedumi 1.02, a matlab toolbox for optimization over symmetric cones. Optimization Methods and Software, 11(1-4), 625-653.

Gomide, T.S. e Lacerda, M.J. (2018). Stability analysis of discrete-time switched systems under arbitrary switching. In IFAC-PapersOnLine, volume 51, 371 - 376.

Hespanha, J.P. e Morse, A.S. (1999). Stability of switched systems with average dwell-time. In Stability of switched systems with average dwell-time, volume 3, 2655-2660 vol.3.

Hetel, L., Daafouz, J., e Iung, C. (2006a). Robust stability analysis and control design for switched uncertain polytopic systems. IFAC Proceedings Volumes, 39(9), 166-171.

Hetel, L., Daafouz, J., e Iung, C. (2006b). Stabilization of arbitrary switched linear systems with unknown time-varying delays. IEEE Transactions on Automatic Control, 51(10), 1668-1674.

Jungers, R.M., Ahmadi, A.A., Parrilo, P.A., e Roozbehani, M. (2017). A characterization of Lyapunov inequalities for stability of switched systems. IEEE Transactions on Automatic Control, 62(6), 3062-3067.

Kermani, M. e Sakly, A. (2014). On stability analysis of discrete-time uncertain switched nonlinear time-delay systems. Advances in Difference Equations, 2014(1), 233.

Khalil, H.K. (2002). Nonlinear Systems. Prentice Hall, United States of America.

Lee, J.W. e Dullerud, G.E. (2006). Uniform stabilization of discrete-time switched and markovian jump linear systems. Automatica, 42(2), 205-218.

Liberzon, D. (2003). Switching in Systems and Control. Birkhauser Boston, Boston.

Liberzon, D. e Morse, A.S. (1999). Basic problems in stability and design of switched systems. IEEE Control Systems Magazine, 19(5), 59-70.

Löfberg, J. (2004). Yalmip : A toolbox for modeling and optimization in matlab. In In Proceedings of the CACSD Conference. Taipei, Taiwan.

Niamsup, P. e Rajchakit, G. (2013). New results on robust stability and stabilization of linear discrete-time stochastic systems with convex polytopic uncertainties. Journal of Applied Mathematics, 2013, 10.

Oliveira, R.C.L.F. e Peres, P.L.D. (2007). Parameter-dependent LMIs in robust analysis: Characterization of homogeneous polynomially parameter-dependent solutions via LMI relaxations. IEEE Transactions on Automatic Control, 52(7), 1334-1340.

Pessim, P.S.P., Lacerda, M.J., e Agulhari, C.M. (2018). Parameter-dependent Lyapunov functions for robust performance of uncertain systems. In Joint 9th IFAC Symposium on Robust Control Design and 2nd IFAC Workshop on Linear Parameter Varying Systems, 441-446. Florianópolis, SC, Brazil.

Pessim, P.S.P., Leite, V.J.S., e Lacerda, M.J. (2019). Robust performance for uncertain systems via Lyapunov functions with higher order terms. Journal of the Franklin Institute, 356(5), 3139-3156.

Rajchakit, G., Rojsiraphisal, T., e Rajchakit, M. (2012). Robust stability and stabilization of uncertain switched discrete-time systems. Advances in Difference Equations, 2012(1), 134.

Sun, Y.G., Wang, L., e Xie, G. (2006). Delay-dependent robust stability and stabilization for discrete-time switched systems with mode-dependent time-varying delays. Applied Mathematics and Computation, 180(2), 428 - 435.

Vidyasagar, M. (1993). Nonlinear Systems Analysis. Prentice Hall.

Zhai, G., Hu, B., Yasuda, K., e Michel, A.N. (2000). Stability analysis of switched systems with stable and unstable subsystems: an average dwell time approach. In Proceedings of the 2000 American Control Conference, volume 1, 200204 vol.1.

Zhang, Y. e Yan, P. (2015). Delay-dependent stability analysis for uncertain switched time-delay systems using average dwell time. Mathematical Problems in Engineering, 2015, 1-8. 